\title{
Niveles de resiliencia en adolescentes de hogares disfuncionales y funcionales en la Unidad Educativa Fiscal Mixta "Pedro Zambrano Barcia"
}

\section{Levels of resilience in adolescents in dysfunctional and functional homes in the Public Educational Unit "Pedro Zambrano Barcia"}

\section{Resiliencia en adolescentes de hogares disfuncionales y funcionales}

\author{
Anicia Tarazona Meza, MSc (1) \\ Inger Maitta Rosado, MSc (2) \\ Miguel Ormaza Cevallos. MBA (3) \\ Dolores Saltos Zamora (4)
}

(1) Universidad Técnica de Manabí, Facultad de Ciencias Humanísticas y Sociales, Magister en Gerencia Educativa, Manabí-Ecuador, atarazona@utm.edu.ec

(2) Universidad Técnica de Manabí, Facultad de Ciencias Humanísticas y Sociales, Magister en Gerencia Educativa, Manabí-Ecuador, imaitta@utm.edu.ec

(3) Universidad Técnica de Manabí, Facultad de Ciencias Administrativas y Económica, Magister en Administración de Empresas, Manabí-Ecuador, mormaza@utm.edu.ec

(4) Estudiante de la carrera de Psicología Clínica de la Facultad de Ciencias Humanística y Sociales, UTM, doloressaltos73@gmail.com

Contacto: doloressaltos73@gmail.com

Recibido: 18-02-2020 Aprobado: 28-02-2020

\section{Resumen}

El objetivo de este estudio es identificar: los niveles de resiliencia en adolescentes de hogares disfuncionales y funcionales. Para ello, se encuestaron a 66 estudiantes de $10^{\circ}$ año de la Unidad Educativa Fiscal Mixta "Pedro Zambrano Barcia", a través de la aplicación de dos cuestionarios: Análisis de la Resiliencia en Adolescentes "A.R.A" y Resilience Scale "RS". Asimismo, la investigación contó con un diseño metodológico cuali-cuantitativo, descriptivo y comparativo, cuyos resultados obtenidos indican que los adolescentes de hogares tanto disfuncionales como funcionales mantienen una moderada resiliencia.

Palabras clave: Resiliencia, adolescentes, hogares disfuncionales y funcionales

\footnotetext{
Abstract

The objective of this study is to identify: the levels of resilience in adolescents of dysfunctional and functional homes. Therefore, a study was conducted to 66 students from the 10th years in the Public Educational Unit "Pedro Zambrano Barcia", through the application of two tests: Analysis of Resilience in Adolescents "A.R.A" and Resilience Scale "RS". Also, the research had a qualitative, quantitative,
}

descriptive and comparative methodological design, which indicate that adolescents from both, it was identified that adolescents from both dysfunctional and functional homes maintain a moderate resilience.

Keywords: Resilience, adolescents, dysfunctional and functional homes.

\section{Introducción}

La resiliencia está vinculada al empoderamiento, autonomía y desarrollo de las diferentes etapas de la vida de un sujeto, por ende, las personas resilientes saben que, aunque su vida está muy marcada, dependen más de sus decisiones, que de los condicionantes, y apuestan de manera decidida por la proactividad (Forés y Grané, 2012). Siendo la resiliencia, el resultado de una serie de acontecimientos ocurridos y no una reflexión conceptual.

De ahí, que en la actualidad los adolescentes durante su etapa escolar se enfrentan a dos escenarios: hogares disfuncionales o funcionales. Por lo que, es preciso señalar, la importancia de la resiliencia en los hogares en situación de riesgo, dado que, si el adolescente, es capaz de mantener la calma, de reflexionar y de cambiar de aptitud, ante las dificultades de la vida familiar y la vida escolar, se 
puede afirmar que mantiene una actitud resiliente, por el contrario, si su reacción es reactiva y comienza a agredir o se paraliza, está lejos de la resiliencia (De Barbieri, 2015). Es así, que lo vivido en el entorno familiar o escolar es algo que frena o que empuja al adolescente a seguir adelante.

De forma más puntual UNICEF menciona, que para la construcción de la resiliencia en adolescentes, se deben entender los fenómenos que constituyen a las amenazas de origen natural, tecnológicos o social e identificar las vulnerabilidades a las que están expuestos (UNICEF, 2013). Por ende, es fundamental garantizar a los adolescentes su participación activa como agente de cambio, durante y después de una situación de riesgo, ya que son un grupo etario altamente sensible, debido a sus necesidades psicoafectivas, físicas y sociales.

En esta misma línea, en América Latina se han reconocido tres cambios importantes en la estructura familiar que ponen en riesgo su funcionamiento: el aumento de las familias monoparentales, altas tasas de fecundidad adolescente y los procesos migratorios. Desde este panorama, los sistemas educativos de la región enfrentan los siguientes desafíos para fortalecer la resiliencia juvenil: los altos niveles de deserción, especialmente en la secundaria, los déficits en la calidad de la educación y la falta de oportunidades de inserción laboral. (PNUD, 2013)

Referente a ello, el Ministerio de Educación junto a UNICEF, Plan Internacional y la Unión Europea han diseñado el proyecto "Construyendo Comunidades Educativas Resilientes en Ecuador", para la disminución de riesgos de las comunidades educativas, mediante estrategias a favor de los niños y adolescentes frente a los sucesos desfavorables de origen natural y antrópicos que puedan exteriorizarse en el entorno escolar.

Por consiguiente, la Constitución de la República de Ecuador, artículo 389, establece que “el Estado protegerá a las personas, colectividades y la naturaleza de los efectos negativos de los desastres de origen natural o antrópico mediante la prevención ante el riesgo, la mitigación de desastres, la recuperación y mejoramiento de las condiciones sociales, económicas y ambientales, con el objeto de minimizar la condición de vulnerabilidad" (Constitución de la República del Ecuador, 2008).

De manera que, el estado de derecho y seguridad ciudadana son elementos claves de la constitución ecuatoriana, mediante un sistema de justicia enfocado en medidas preventivas y políticas de seguridad, que contribuyen al fortalecimiento de los hogares en situaciones de riesgo, no obstante, las relaciones familiares son aún más compleja, es así, que en la provincia de Manabí, se puedo evidenciar que en el año 2018, por cada 1.000 mujeres, el 3,68 de nacimientos, corresponden a madres adolescentes de 10 a 14 años, a esto se suma, el 86,73 de tasa de nacimientos de madres adolescentes de 15 a 19 años (Registro Estadístico Nacidos Vivos, 2018).

Por ende, es necesario construir entornos familiares resilientes con niveles óptimos de equilibrio y bienestar, para superar crisis y sucesos negativos que conllevan a las familias a situaciones de riesgo. No obstante, son pocos los estudios centrados en los niveles de resiliencia en adolescentes de hogares disfuncionales y funcionales, la mayoría de los trabajos están enfocados en la construcción de resiliencia en hogares vulnerables, ante fenómenos que constituyen una amenaza de origen natural.

De ahí, la necesidad de profundizar en la dinámica de los hogares disfuncionales y funcionales, a través un enfoque sociohistórico y sociodemográfico, en virtud de la información estadística disponibles en test y encuestas, para describir los diversos niveles $\mathrm{o}$ aspectos de resiliencia que los vinculan.

Por lo expuesto anteriormente, el objetivo de la investigación es identificar los niveles de resiliencia en adolescentes de hogares disfuncionales y funcionales en la Unidad Educativa Fiscal Mixta "Pedro Zambrano Barcia"

\section{Niveles de resiliencia en adolescentes de hogares disfuncionales y funcionales.}

El término resiliencia encierra variadas aproximaciones que integran elementos de carácter individual, social, familiar y escolar, las que están relacionadas con factores de riesgo, vulnerabilidad y protección. Es una nueva concepción del individuo que pasa de estar desvalido, a considerarse capaz de enfrentarse a las adversidades e inclusive de tomar de nuevo las riendas de su propia vida (Puig y Rubio, 2011). Estas características se definen a partir de la capacidad del individuo de adaptarse saludablemente en situaciones infortunas.

Es así, que la fuerza psíquica de un individuo y de cómo se adapte, resista o afronte situaciones hostiles que se presentan en su diario convivir; se le denomina resiliencia, la cual ayuda a las personas a superar situaciones complejas (Tarazona, 2019). Por lo tanto, la persona resiliente tiene la capacidad de enfrentar entornos problemáticos, de esta manera, le ayudará en su fortalecimiento individual.

Desde el punto de vista social, es un concepto entendido como un proceso, en el que median variables personales, que se forjan desde lo social, lo relacional y los ecosistemas humanos (Madariaga, 2014). Entendiéndose a la resiliencia como el resultado de la interacción social del individuo con el 
grupo al que pertenece, el cual se construye en la comunidad en la que vive.

En este sentido, la familia es el principal factor de protección para que el niño o adolescente se pueda enfrentar a acontecimientos adversos, un entorno familiar seguro, afectuoso y estable, permite el desarrollo de comportamientos resilientes. Sin embargo, en la actualidad las familias se enfrentan a situaciones cada vez más complejas: el incremento de madres solteras, la migración, la disminución de autoridad en los roles paternos, divorcios, causan el aumento de hogares disfuncionales.

¿Cuándo un padre es maltratador? ¿Exigir disciplina es sinónimo de maltrato? Datos estadísticos demuestran que cuando los dos padres maltratan al niño, se detecta el $90 \%$ de niños inseguros, cuando el maltratador solo es uno de los padres, se detecta el $60 \%$ de niños inseguros e incluso hasta en hogares funcionales se encuentran el 30\% de niños inseguros. Por lo tanto, se puede hablar de resiliencia familiar cuando, después de un suceso inesperado, la familia se distancia del sufrimiento y transforma el trauma en relato del pasado y en actividades que pueden compartir con personas de su entorno (Cyrulnik y Anaut, 2016).

Al igual que con los padres, para conseguir el éxito en la resiliencia de los niños y adolescentes es necesario que el docente esté involucrado, ya pasan gran parte del día en la institución educativa, siendo el docente la figura referente con quien establecen relaciones de afecto, con una adecuada formación docente, se puede desarrollar actitudes que permitan el fortalecimiento de la resiliencia de acuerdo a las necesidades de cada niño y adolescentes.

Por ende, una educación de calidad, lo será en la medida en que la resiliencia sea abordada en el currículo, desde la educación inicial hasta los planes de estudios universitarios, con contenidos relativos a las necesidades de los estudiantes en situación de riesgo y vulnerabilidad, a través de un proceso de auto aprensión, a partir de experiencias personales, de modo que, no solo se considere el desarrollo de las capacidades cognitivas y emocionales, sino, sobre todo, lo vivido en su entorno físico y social.

Asimismo, es importante señalar los elementos educativos para fomentar la resiliencia en niños, adolescentes y jóvenes.

- Conocer la realidad: aumenta la resiliencia en los adolescentes, pues adquieren la capacidad de discernir, a través de respuestas honestas de las personas adultas que están en su entorno.

- Independencia, participación y autonomía: se establece mediante la capacidad de los adultos de reconocer en los adolescentes la habilidad de orientarse en base a sus necesidades creando un vínculo entre la escuela y familia.

- Ampliar la posibilidad de establecer relaciones con el entorno social: establece una red positiva de relaciones sociales $y$ potencia la protección ante situaciones de riesgo.

- Explorar todo el entorno: el objetivo es adquirir la capacidad de resolver los problemas difíciles.

- Mantener la capacidad de jugar: los niños, adolescentes, jóvenes y adultos, que saben jugar, adquieren la capacidad de resolver los problemas.

- Educar la capacidad de juzgar y desarrollar el sentido crítico: los niños y adolescentes necesitan una educación ética de calidad, repensar los valores que recibieron de sus padres y considerar el servicio a los demás como forma de compromiso social (Sánchez, et al, 2015)

Otra forma de fomentar los elementos internos de la resiliencia en los niños y adolescentes es a través de los cuentos, dibujos animados, películas y libros, los estudiantes pueden crear sus propios personajes, identificarse con algunas características e ir desarrollando conciencia del problema en el que encuentran, adentrándose hacia un futuro positivo y ser capaces de entenderse a sí mismos.

Se ha abordado las etapas niñez y adolescencia, en el estudio se hace énfasis en la adolescencia que depende en última instancia de una niñez feliz Por consiguiente, es importante resaltar los diversos enfoques relacionados con el estudio de la adolescencia, que han surgido a lo largo del tiempo con el fin de explicar el comportamiento del adolescente durante este ciclo vital. Este periodo de transición, constituye una etapa de cambios que conduce a la madurez, a través de una serie de transformaciones psicológicas, sociales y culturales (Moreno, 2015).

Al respecto, estudios realizados en México en resiliencia con adolescentes de ambientes de marginación y pobreza, muestran que el $84 \%$ de los padres de adolescentes son jóvenes con un rango de edad de 30 y 45 años, en cuanto al nivel de instrucción el padre presenta un mayor grado de escolaridad que la madre, el $65 \%$ de los padres son obreros y el $50 \%$ de las madres son amas de casa, el $50 \%$ de las familias son nucleares, el $28 \%$ son monoparentales, el $15 \%$ son extensas y el $8 \%$ son reconstruidas. Todos estos indicadores resultaron significativos para identificar que a pesar de la pobreza o adversidad económica los adolescentes, 
mostraron adaptación, es decir son resilientes (Barcelata, 2015).

En tal sentido, la resiliencia en los adolescentes se va estructurando a partir de la niñez y la interacción con el entorno, esencialmente de índole familiar, lo que influye tanto, en el desarrollo correcto del comportamiento como en el comportamiento inadecuado del adolescente. Los hogares funcionales permiten que se generen soluciones ante diversos problemas personales de la vida, sin embargo, los hogares disfuncionales pueden conllevar al adolescente a conductas peligrosas que requieran de ayuda profesional.

De hecho los hogares disfuncionales se caracterizan por la ausencia de comunicación, de preocupación y control, abandono, maltrato físico y emocional, necesidades básicas insatisfechas, presencia de adicciones entre otros (Kalbermatter, Goyeneche y Heredia, 2006). Contrario a los hogares funcionales que se definen como un núcleo de convivencia donde se cumplen las condiciones mínimas para que sus miembros puedan desarrollarse de manera armónica, física, emocional, intelectual y socialmente (Press, 2011).

Contreras (2018) precisa que aquellos hogares en los que las funciones básicas están activas y son altamente efectivas se les denominan funcionales. Así, al hablar de los hogares disfuncionales no existe la expresión afectiva, se percibe el conflicto y no se satisfacen las necesidades básicas (Canales, 2018).

De modo que, los padres y madres de hogares disfuncionales son incapaces de satisfacer las necesidades básicas y emocionales de cada uno de los miembros de su familia, aunque exista la intensión, no comprenden con claridad cómo hacerlo, existiendo una presión interna que suele convertirse en maltrato físico y psicológico. Del mismo modo, los padres y madres de hogares funcionales se caracterizan por sus relaciones de armonía, la comunicación es directa, la organización jerárquica es clara, tienen la capacidad de resolver dilemas a través del diálogo, realizan planes juntos y disfrutan compartiendo en familia.

En síntesis, en estas últimas décadas los hogares a nivel mundial han mostrado cambios muy marcados como divorcios, aumento de madres adolescentes, familias monoparentales con mayor prevalencia de madres jefas del hogar, así como contextos de alto riesgo como pobreza, enfermedad crónica, eventos traumáticos, dichas variables familiares y sociales repercute en los adolescentes. De lo que se concluye que la resiliencia en los adolescentes los conduce a mejorar su calidad de vida, a partir de la manera de cómo perciben y enfrentan situaciones de adversidad.

\section{Materiales y métodos}

La investigación tuvo un enfoque cuali-cuantitativo, descriptivo y comparativo. Se utilizó un cuestionario elaborado por la autora con variables sociodemográficas, para determinar el tipo de familia a la que pertenecen los adolescentes de la Unidad Educativa Fiscal Mixta "Pedro Zambrano Barcia".

Asimismo, se aplicó el cuestionario A.R.A “Análisis de la Resiliencia en Adolescentes" validado en Colombia, construido por Jessica Jabela, María Rocha, Ángela Serna en el año 2014. A través del cuestionario se identificaran las características que los adolescentes tienen respecto a la resiliencia. Está compuesta por 30 ítems, los cuales puntúan en escala tipo Likert de 7 puntos, donde 1 es máximo de acuerdo, y 7 máximo desacuerdo. Los participantes indicarán el grado de conformidad con la afirmación, su administración es individual con una duración de 15 a 20 minutos aproximadamente.

Para la construcción del cuestionario "ARA" se tomó en consideración la Escala de Resiliencia de (RS) de Wagnild y Young (1993) que fue elaborada para identificar el grado de resiliencia individual de adolescentes y adultos. Consta de 25 reactivos que oscilan desde $1=$ totalmente en desacuerdo, a 5 totalmente de acuerdo, son puntuados en una escala con formato tipo Likert de 7 puntos, siendo todos los ítems calificados positivamente, fluctuando los puntajes entre 25 a 175 puntos.

La población que se tomó como base de la investigación estuvo compuesta por 66 estudiantes de $10^{\circ}$ año de "EGB" de la Unidad Educativa Fiscal Mixta "Pedro Zambrano Barcia", tomando como muestra él $100 \%$ de su totalidad, se utilizó el muestreo probabilístico, garantizando que cada individuo tenga las mismas oportunidades de ser seleccionado mediante la aleatorización.

\section{Resultados}

A continuación se presenta los resultados cuantitativos y estadísticos obtenidos en el proceso de la encuesta aplicada a los estudiantes de $10^{\circ}$ año de "EGB" de la Unidad Educativa "Pedro Zambrano Barcia”, para el seguimiento sociodemográfico y análisis del tipo de familia a la que pertenecen los adolescentes de la institución.

\section{Lugar de residencia}

Análisis: Se observa que 53 adolescentes que equivale al $80 \%$ residen en el área urbana, mientras que 13 adolescentes que equivale al $20 \%$ residen en el área rural.

\section{¿Tu papá y tu mamá viven juntos?}


Análisis: Los resultados demuestran que 40 adolescentes que representa el $61 \%$ sus padres viven juntos. Sin embargo, 26 adolescentes que representa el 39\% sus padres están separados, lo cual refleja la ausencia de alguno de los padres, presentando un porcentaje significativo de hogares disfuncionales.

\section{¿Quiénes integran tu familia?}

Análisis: Se evidencia que 14 adolescentes de hogares disfuncionales que equivale al $54 \%$ conviven con mamá y hermanos, dos adolescentes que equivale al $8 \%$ con papá y hermanos, siete adolescentes que equivale al $27 \%$ con abuelos y tíos, dos adolescentes que equivale al $8 \%$ con papá y madrastra y un adolescente que equivale al $4 \%$ convive solo con su papá. Referente a los adolescentes de hogares funcionales, 33 adolescentes que equivale al $83 \%$ manifestaron que su familia está conformada por mamá, papá y hermanos y 7 adolescentes que equivale al $18 \%$ está conformada por mamá y papá. Evidenciando, la ausencia de por lo menos un miembro de la unidad familiar en los hogares disfuncionales.

\section{Relación con tus padres}

Análisis: Se observa que seis adolescentes de hogares disfuncionales que representa el 23\% la relación con sus padres es buena, cinco adolescentes que representa el $19 \%$ es mala, 15 adolescentes que representa el $58 \%$ es regular, a comparación de los adolescentes de los hogares funcionales, 35 adolescentes que representa el $88 \%$ la relación es buena y 5 adolescentes que representa el $13 \%$ es regular, reflejando que los adolescentes de hogares funcionales en su mayoría mantienen una buena relación con sus progenitores.

\section{Nivel de Calificación}

Análisis: Se pudo comprobar que cuatro adolescentes de hogares disfuncionales que equivale al $15 \%$ el nivel de calificación es excelente, 16 adolescentes que equivale al $62 \%$ es poco satisfactorio, seis adolescentes que equivale al $23 \%$ es nada satisfactorio. A diferencia de los adolescentes de hogares funcionales, 25 adolescentes que equivale al $63 \%$ el nivel de calificación es excelente, 15 adolescentes que equivale al $38 \%$ es poco satisfactorio.

\section{Tus padres se caracterizan por:}

Análisis: Los datos obtenidos evidencian que dos adolescentes de hogares disfuncionales que representa el $8 \%$ sus padres se caracterizan por ser sobreprotectores, ocho adolescentes que representa el $31 \%$ son liberales, 10 adolescentes que representa el $38 \%$ son despreocupados, cinco adolescentes que representa el 19\% son preocupados y solo un adolescente representado por el $4 \%$ contestó que sus padres son muy cuidadosos. A comparación de los padres de hogares disfuncionales, 15 adolescentes de hogares funcionales que representa el $38 \%$ sus padres se caracterizan por ser sobreprotectores, siete adolescentes que representa el $18 \%$ muy conservadores, nueve adolescentes que representa el $23 \%$ son preocupados, seis adolescentes que representa el $15 \%$ muy cuidadoso y tres adolescentes que representa el $8 \%$ contestaron que sus padres se caracterizan estar siempre juntos.

\section{Existe comunicación entre tus padres}

Análisis: En relación con los resultados, seis adolescentes de hogares disfuncionales que representa el $23 \%$ contestaron que entre sus padres existe comunicación, mientras que 20 adolescentes que representa el $77 \%$ contestaron que entre sus padres no existe comunicación. A diferencia de los padres de hogares funcionales, 35 adolescentes que representa el $88 \%$ indicaron que entre sus padres existe comunicación y 5 adolescentes que representa el $13 \%$ indicaron que no existe comunicación entre sus padres.

\section{Análisis de resultados}

A través de la información obtenida en la encuesta aplicada a 66 estudiantes adolescentes de la Unidad Educativa Fiscal Mixta "Pedro Zambrano Barcia", se pudo observar que la mayor parte de los estudiantes reside en la zona urbana, asimismo, se determinó que un grupo significativo de adolescentes de la institución provienen de hogares disfuncionales, cuyos padres están separados, lo cual repercute en una mala relación familiar $\mathrm{y}$ en el nivel de calificaciones de los adolescentes. Además de ello, también existe despreocupación, falta de comunicación y confianza entre los miembros de la familia.

Respecto a los padres de los hogares funcionales, muestran una actitud integradora, lo cual favorece al aprendizaje, el desarrollo emocional y moral de sus hijos adolescentes. Si bien es cierto esta tarea es ardua, y para lograr esta meta, los padres deben proporcionar a sus hijos un ambiente libre de riesgos, para que puedan enfrentar los desafíos de la vida diaria.

Resultado del cuestionario A.R.A "Análisis de la Resiliencia en Adolescentes" de Jessica Jabela, María Rocha y Ángela Serna (2014).

El cuestionario aplicado se centró en determinar el nivel de resiliencia de los estudiantes de hogares disfuncionales y funcionales de la Unidad Educativa Fiscal Mixta "Pedro Zambrano Barcia", cuyas 
unidades de análisis son las siguientes: Ecuanimidad: 6 ítems (2-5-7-17-24-25), Perseverancia: 5 ítems (13-21-22-26-28), Confianza en sí mismo: 9 ítems (46-12-13-14-16-18-19-20), Satisfacción personal: 3 ítems (9-11-23) y Sentirse bien solo: 5 ítems (8-1015-27-29-30). Para poder evaluar el resultado del cuestionario se le determinó un puntaje específico a la escala, de la siguiente manera:

En acuerdo: (1-2-3-4)

En desacuerdo: (5-6-7)

\section{Las experiencias negativas a través de la vida me} han fortalecido

Análisis: Se evidencia que los adolescentes de hogares disfuncionales y hogares funcionales mantienen una aptitud ecuánime ante la adversidad.

\section{Soy capaz de vencer los obstáculos que se me presenta en la vida.}

Análisis: Los adolescentes de hogares disfuncionales son menos constantes que los adolescentes de hogares funcionales.

\section{Cuando tomo decisiones me siento seguro.}

Análisis: Los adolescentes de los hogares disfuncionales y funcionales se sienten capaces de afrontar las dificultades que se les presenta en su entorno.

\section{Considero que llevo una calidad vida buena a pesar de lo sucedido}

Análisis: Los adolescentes de los hogares disfuncionales llevan en menor medida una buena calidad de vida en comparación con los adolescentes de los hogares funcionales.

\section{Entre más lejos este de mi familia, me siento mejor.}

Análisis: La mayoría de los adolescentes de los hogares disfuncionales y hogares funcionales prefieren estar cerca de la familia.

\section{Análisis de resultados.}

Se concluye de manera global al comparar los datos del cuestionario Análisis de la Resiliencia en Adolescentes "A.R.A", de los hogares disfuncionales $\mathrm{y}$ funcionales lo siguiente: $\mathrm{El}$ componente "Ecuanimidad", están "en acuerdo", es decir los adolescentes se han fortalecido ante las experiencias negativas.
Respecto al componente "Perseverancia", se observa que en los hogares donde existe abandono, maltrato, ausencia de amor y respeto, los adolescentes son capaces de vencer dichas adversidades, aunque en menor medida en comparación con adolescentes que conviven en un hogar armónico y equilibrado. Es así, que en el componente "confianza en sí mismo", se evidencian resultados positivos, estudiantes con una personalidad fortalecida que les ayuda a mejorar su desempeño académico y social.

Sin embargo, en el componente "Satisfacción personal", se comprueba que en los hogares en cuyo funcionamiento intrafamiliar se gestan alteraciones negativas, se desencadena una baja calidad de vida, a diferencia del funcionamiento familiar saludable de los hogares donde existe la solidaridad, responsabilidad compartida, la ayuda y el respeto mutuo.

Finalmente, en el componente "sentirse bien sólo" los resultados demuestran que en su mayoría, los adolescentes prefieren estar cerca de su familia, lo cual contribuye a elevar el nivel de resiliencia de los mismos. Por lo tanto, a través de la aplicación del test se determinó que el nivel de resiliencia de los adolescentes de hogares disfuncionales es inferior al de los adolescentes de hogares funcionales.

\section{Discusión}

La resiliencia en la actualidad es considerada como la capacidad humana de enfrentarse, sobreponerse y transformarse después de experiencias difíciles. Es así, que después de un análisis global de la problemática observada, la percepción de hogares disfuncionales es del 39\%, resultado coherente con lo descrito en la investigación realizada por Higuita y Cardona (2014) quienes en su artículo "Percepción de funcionalidad familiar en adolescentes escolarizados en instituciones educativas públicas de Medellín" evidencian que el $30,6 \%$ lo integran las familias disfuncionales. De éstas, el 75,5\% presentan disfuncionalidad moderada y el $24,5 \%$ disfuncionalidad grave.

Antes este contexto Barrios y Verdecia (2016), en su artículo "Las familias disfuncionales como factor de riesgo adictivo en la adolescencia" concluyen que las relaciones familiares se establecen como un factor de riesgo adictivo fundamental para la adolescencia, cuya calidad de vida estará determinada en mayor o menor medida por los índices de maltrato, violencia doméstica, la comunicación deficiente e inconsistencia afectiva.

Aunado a ello, Pérez y Reinoza (2011) en su artículo "El educador y la familia disfuncional" determinan la importancia del rol de la escuela, como ente capaz de dispensar el afecto que en muchas familias está 
ausente. Por ende, es importante fomentar la autoestima y autonomía través del juego, involucrando a todos los miembros de la comunidad, no solo al adolescente, esto implicará potenciar los recursos existentes para el desarrollo de factores resilientes en adolescentes que viven en situaciones de riesgo, trabajando de manera conjunta y coordinada, debido a que no es una tarea que corresponda de manera exclusiva ni a los padres, ni a la escuela, ni al estado, es una tarea y una responsabilidad de todos.

\section{Conclusiones}

Al concluir el trabajo de investigación, se revela que un grupo significativo de adolescentes provienen de hogares disfuncionales, cuyos padres están separados, lo que repercute en una mala relación familiar y en el nivel de calificaciones de los mismos. A diferencia de los padres de los adolescentes de hogares funcionales que protegen en mayor medida a sus hijos, lo que influye en el fortalecimiento de la autoestima, autonomía y participación de los adolescentes en los diferentes ámbitos de su devenir cotidiano (familia-escuela-sociedad).

Asimismo, estos resultados muestran que existe una correlación entre los adolescentes de los hogares disfuncionales y funcionales, quienes a pesar de las dificultades mantienen una moderada resiliencia. Por lo tanto, se logra el objetivo de la investigación que fue: identificar los niveles de resiliencia en adolescentes de hogares disfuncionales y funcionales en la Unidad Educativa Fiscal Mixta "Pedro Zambrano Barcia", siendo la resiliencia, la habilidad que otorga al adolescente las herramientas necesarias para contrarrestar las reflexiones distorsionadas, reducir la ansiedad y tomar decisiones adecuadas.

\section{Bibliografía}

Barcelata, B. (2015). Adolescentes en riesgo: Una mirada a partir de la resiliencia. El Manual Moderno, S.A. de C.V.: Zaragoza.

Barrios, Y. y Verdecia, R. (2016). Las familias disfuncionales como factor de riesgo adictivo en la adolescencia. Adicción y Ciencia. 4 (1). Consultado 20 de noviembre de 2019. Recuperado de: http://www.codajic.org/sites/www.codajic.or g/files/Las \%20familias\%20disfuncionales $\% 2$ 0como $\% 20$ factor $\% 20$ de $\% 20$ riesgo $\% 20$ adicti vo\%20en\%201a\%20adolescencia\%20\%20.pd $\underline{f}$

Canales, J. (2018). Padres tóxicos: legado disfuncional de una infancia tóxica. Páidos: México.
Contreras, R. (2018). Un hombre con un mundo sin sentido: Un Legado a la siguiente generación. Westbow Press.

Cyrulnik, B., y Anaut, M. (2016). ¿Por qué la resiliencia? Lo que nos permite reanudar la resiliencia. Gedisa: Barcelona.

De Barbieri, A. (2015). Educar sin culpas. Optimismo y entusiasmo para padres $y$ docentes. Gribaldo: Panamá.

Forés, A., y Grané, J. (2012). La resiliencia en entornos socioeducativos. Sentidos, propuestas y experiencias. Marcea, S.A.: Madrid

Higuita, L.F. y Cardona, J. (2016). Percepción de funcionalidad familiar en adolescentes escolarizados en instituciones educativas públicas de Medellín. CES Psicología. 9(2), 167-178. Consultado 205de noviembre de $2019 . \quad$ Recuperado de: http://www.scielo.org.co/pdf/cesp/v9n2/2011 -3080-cesp-9-02-00167.pdf

Instituto Nacional de Estadísticas y Censo del Ecuador, INEC, (2018). Registro Estadístico Nacidos Vivos y Defunciones.

Kalbermatter, M., Goyeneche, S., y Heredia, R. (2006) Resiliente se nace, se hace, se rehace. Brujas: Córdova.

Madariaga, J. (2014). Nuevas miradas sobre la resiliencia. Ampliando ámbitos y prácticas. Gedisa, S.A.: Barcelona.

Moreno, A. (2015). La adolescencia. UOC, SL: Barcelona

Pérez, A. y Reinoza, M. (2011) El educador y la familia disfuncional. Educere, 15(52), 629634. Consulta 12 de Diciembre de 2019. Recuperado de: https://www.redalyc.org/articulo.oa?id=3 $\underline{5622379009}$

Press, E. (2011). Empresas de familias: Del conflicto a la eficiencia. Gramica S.A. Bueno Aires.

Programa de las Naciones Unidas para el Desarrollo, PNUD, (2013). Informe Regional de Derecho Humano 2013-2014, Seguridad Ciudadana con Rostro Humano: Diagnóstico y Propuesta para América Latina. Centro Regional de Servicios para América Latina y el Caribe Dirección Regional para América Latina y el Caribe Programa de las Naciones Unidas para el Desarrollo (PNUD): Panamá. 
Puig, G., y Rubio, J. (2011). Manual de resiliencia aplicada. Gedisa S.A.: Barcelona

Sánchez, C., Medina, A., Domingo, J., Rodríguez, R., Almenar, N., Escudero, N., Muñoz, V., Sánchez, A., Alonso, J., Gutiez, P., Moratalla, S., Lago, P. (2015). Formación y Desarrollo Profesional del Ecuador en Contextos de Intervención. Universidad Central de Educación a Distancia.

Tarazona, k. (2019). Resiliencia en pacientes drogodependientes de la Comunidad Terapéutica "Camino a La Paz" Espirales, 3 (30.614), 26-42. 10.31876. Recuperado de: https://www.researchgate.net/publication/334 464094_Resiliencia_en_pacientes_drogodep endientes_de_la_Comunidad_Terapeutica_C amino_a_La_Paz

UNICEF (2013). Acciones para la resiliencia de la niñez y adolescentes. JEICOS S.A.: Panamá. 